\title{
Sub-50 fs All-fiber Yb-Doped Laser with Anomalous-Dispersion Photonic Crystal Fiber
}

\author{
Z. Zhang, ${ }^{1}$ Ç. Şenel, ${ }^{1,2}$ R. Hamid, ${ }^{2}$ and F. Ö. Ilday ${ }^{1}$ \\ ${ }^{1}$ Department of Physics, Bilkent University, 06800 Ankara, Turkey \\ ${ }^{2}$ TÜBITAK National Metrology Institute (UME), 41470 Kocaeli, Turkey
}

An intense research effort has been channelled into improving mode-locked Yb-fiber oscillators in recent years. Despite efforts in all-normal dispersion oscillators, dispersion management is evidently necessary to reach pulse durations below $50 \mathrm{fs}$. This is implemented most commonly with bulk optical components in Yb-doped fiber lasers. Increased robustness remains a valuable trait, for which all-fiber-integration is highly desirable. Photonic crystal fibers (PCF) with anomalous dispersion have small mode field diameters, enhancing nonlinear effects and usually are birefringent. The first mode-locked laser to incorporate a PCF was reported in 2002 [1]. However, modelocking was not self-starting owing to the residual birefringence of the PCF. Since then, a number of dispersionmanaged Yb-doped fiber lasers using PCFs and all-fiber-integrated lasers have been reported. After 10 years, no all-fiber-integrated $\mathrm{Yb}$-fiber laser has been demonstrated to support pulses below $60 \mathrm{fs}$ [2].

Here, we present an all-fiber-integrated, dispersion-managed $\mathrm{Yb}$-doped oscillator incorporating a segment of PCF. The oscillator is self-starting and generates pulses with a spectral bandwidth of $58.2 \mathrm{~nm}$, compressible externally to 42 fs. The pulse evolution had to be carefully designed to achieve this performance: the residual birefringence that prevented self-starting operation of the first fiber laser with PCF [1] is actively employed to construct an intra-cavity Lyot filter to ease initiation of mode-locking [3]. The filtering effect has been characterized using a Sagnac loop seeded by a broadband amplified emissions source (Fig. 1(a)). Numerical simulations were performed to investigate the mode-locking dynamics (Fig. 1(b)). The pulse duration and spectral bandwidth decrease in the first half of the gain fiber, then grow essentially self-similarly. Initial compression in the PCF is followed by stretching, accompanying with spectral broadening and narrowing. The pulse energy is reduced to $\sim 78 \mathrm{pJ}$ in the PCF to prevent excessive nonlinear effects. This requires an intra-cavity gain of 60 to reach the maximum pulse energy of $4.7 \mathrm{~nJ}$. While the overall evolution is consistent with stretched-pulse operation, influence of self-similar evolution in the gain fiber is observed due to the strong nonlinearity, which counteracts gain narrowing.
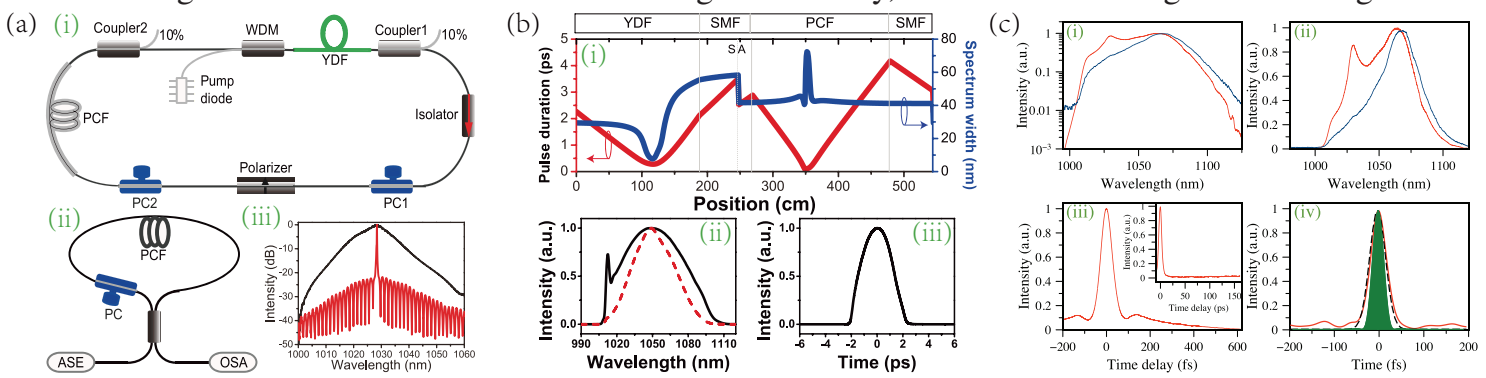

Fig. 1 (a) (i) Schematic of the setup. (ii) Sagnac loop constructed to measure the birefringence of the PCF. (iii) Spectrum of the ASE signal entering the Sagnac loop (black line) and the spectrum of the signal transmitted through the Sagnac loop (red line). (b) Simulated results: (i) Evolution of the spectral (blue line) and temporal (red line) width as a function of position along the cavity. (ii) Spectra after the YDF (solid line) and after the PCF (dashed line). (iii) Pulse shape after the YDF. (c) Experimental results: Spectra measured after YDF (red, solid line), before WDM (blue, dotted line) on (i) logarithmic scale and (ii) linear scale. (iii) Autocorrelation traces measured after the gain fiber and dechirping. Inset shows autocorrelation of chirped pulses. (iv) Pulse shape retrieved using PICASO algorithm (red, solid line) with a Gaussian fit (black, dashed line), and transform-limited pulse (olive, filled line).

In conclusion, we have demonstrated an all-fiber-integrated $\mathrm{Yb}$-doped laser with an anomalous-dispersion PCF. The compressed pulse duration is $42 \mathrm{fs}$, which is the shortest, to the best of our knowledge, from an all-fiber oscillator at $1 \mu \mathrm{m}$. These results have been achieved 10 years after the first mode-locked oscillator with PCF was demonstrated, which was plagued with non-self-starting operation and limited long-term stability primarily due to the residual birefringence of the PCF. Here, the birefringence of the PCF is exploited to function as a fiberintegrated Lyot filter for stable and self-starting mode-locking. Nonlinear effects and spectral shaping throughout the cavity is carefully managed to avoid multiple pulsing.

\section{References}

[1] H. Lim, F. Ö. Ilday, F. W. Wise, “Femtosecond ytterbium fiber laser with photonic crystal fiber for dispersion control,” Opt. Express 10, 1497 (2002).

[2] M. Schultz, O. Prochnow, A. Ruehl, D. Wandt, D. Kracht, S. Ramachandran, and S. Ghalmi, "Sub-60-fs ytterbium-doped fiber laser with a fiber-based dispersion compensation," Opt. Lett. 32, 2372 (2007).

[3] K. Özgören, F. Ö. Ilday, “A filterless all-fiber all-normal dispersion laser,” Opt. Lett. 35, 1296 (2010). 